\title{
Global Dynamics of an Exploited Prey-Predator Model with Constant Prey Refuge
}

\author{
Uttam Das, ${ }^{1}$ T. K. Kar, ${ }^{2}$ and U. K. Pahari ${ }^{1}$ \\ ${ }^{1}$ Department of Mathematics, Sree Chaitanya College, Habra, North 24 Parganas 743268, India \\ ${ }^{2}$ Department of Mathematics, Bengal Engineering and Science University, Shibpur, Howrah 711103, India
}

Correspondence should be addressed to Uttam Das; uttam_das76@yahoo.in

Received 27 May 2013; Accepted 8 July 2013

Academic Editors: M. T. Figge, M. José, A. A. Polezhaev, and J. H. Wu

Copyright (C) 2013 Uttam Das et al. This is an open access article distributed under the Creative Commons Attribution License, which permits unrestricted use, distribution, and reproduction in any medium, provided the original work is properly cited.

\begin{abstract}
This paper describes a prey-predator model with Holling type II functional response incorporating constant prey refuge and harvesting to both prey and predator species. We have analyzed the boundedness of the system and existence of all possible feasible equilibria and discussed local as well as global stabilities at interior equilibrium of the system. The occurrence of Hopf bifurcation of the system is examined, and it was observed that the bifurcation is either supercritical or subcritical. Influences of prey refuge and harvesting efforts are also discussed. Some numerical simulations are carried out for the validity of theoretical results.
\end{abstract}

\section{Introduction}

Prey-predator models are of great interest to researchers in mathematics and ecology because they deal with environmental problems such as community's morbidity and how to control it and optimal harvest policy to sustain a community. In the physical sciences, generic models can be constructed to explain a variety of phenomena. However, in the life sciences, a model only describes a particular situation. So a variety of models are needed due to the complexity of the ecosystem. Theoretical and numerical studies of these models are able to give us an understanding of the interactions that are taking place.

While investigating biological phenomena, there are many factors which affect dynamical properties of biological and mathematical models. One of the familiar nonlinear factors is the functional response. In population dynamics, a functional response of the predator to the prey density refers to the change in the density of prey attached per unit time per predator as the prey density changes [1]. Holling [2] suggested three different kinds of functional response for different kinds of species to model the phenomena of predation, which made the classical Lotka-Volterra system more realistic. The other factor which affects dynamical properties is harvesting. The subject of harvesting in predator-prey systems has been of interest to economists, ecologists, and natural resource managers for some time now. There are basically three types of harvesting reported in the literature: (i) constant harvesting where a constant number of individuals are harvested per unit time, (ii) proportional harvesting where the number of individuals harvested per unit time is proportional to the current population, and (iii) nonlinear harvesting. Most research has focused attention on optimal exploitation, guided entirely by profits from harvesting. First of all, depending on the nature of the applied harvesting strategy, the long-run stationary density of population may be significantly smaller than the long-run stationary density of a population in the absence of harvesting. Therefore, while a population can, in the absence of harvesting, be free of extinction risk, harvesting can lead to the incorporation of a positive extinction probability and therefore, to potential extinction in finite time. Secondly, if a population is subjected to a positive extinction rate, then harvesting can drive the population density to a dangerously low level at which extinction becomes a sure thing no matter how the harvester affects the population afterwards.

Effects of harvesting on various types of prey-predator models have been considered by many researchers. Mathematical modeling with harvesting renewable resources started with the studies of Clark [3, 4]. Xiao et al. [5] have investigated the dynamics of a system with constant 

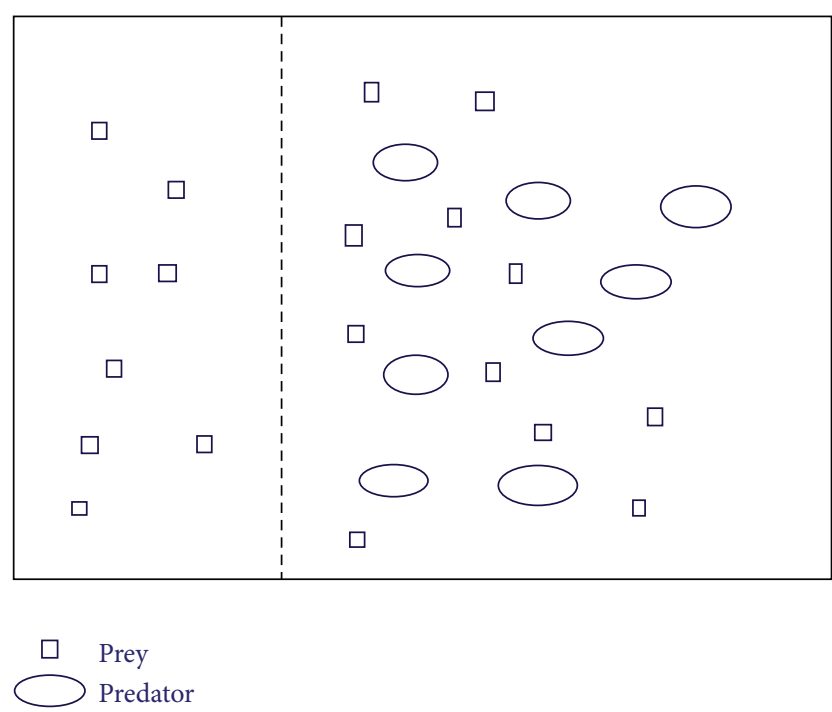

FIgURE 1: Conceptual diagram of the Rosenzweig-MacArthur model with prey refuge. Small quadrilateral and oval shapes represent the prey and predator, respectively. The vertical dashed line represents the boundary of a refuge, where prey species are invulnerable to predators. The refuge could protect either a constant number or a constant fraction of the total prey population.

harvesting on the predator, whereas Leard et al. [6] have studied the dynamics of ratio-dependent models that include nonconstant harvesting on the prey. Xia et al. [7] studied the combined effects of harvesting and time delay. Recently, Pahari and Kar [8] analyzed a prey-predator fishery model with harvesting where they applied a tax to regulate the fishery.

The existence of prey refuges can clearly have important effects on the coexistence of predators and their prey. According to Taylor [9], the different kinds of refuges can be arranged into three types: (a) those which provide permanent spatial protection for a small subset of the prey population, (b) those which provide temporary spatial protection, and (c) those which provide a temporal refuge in numbers, that is, decrease the risk of predation by increasing the abundance of vulnerable prey. It is also observed that refuge has a stabilizing effect on the equilibrium for a simple LotkaVolterra model. The problem of predator-prey interactions under a prey refuge has been studied by some authors. McNair [10] showed that several kinds of refuges could exert a locally destabilizing effort and create stable, large-amplitude oscillations which would damp out if no refuge was present. McNair [11] obtained that a prey refuge with legitimate entry existing dynamics was quite capable of amplifying rather than damping predator-prey oscillation. Kar [12] proposed a predator-prey model incorporating a prey refuge and independent harvesting on either species. He showed that, using the harvesting efforts as control, it is possible to break the cyclic behavior of the system. Wang et al. [13] considered a prey-predator system where individuals from a prey fish population could hide in holes where predators were unable to enter. Ji and $\mathrm{Wu}$ [14] considered a predator-prey model incorporating a constant prey refuge and a constant-rate prey harvesting and showed that the system is controlled by using constant harvesting or prey refuge. Prepredator interactions with prey refuge also may be found in the work of Huang et al. [15], Wang and Pan [16], Kar et al. [17], and so forth.

The objective of this paper is to study the combined effects of harvesting and constant prey refuge on the dynamics of predator-prey model. We also examined the existence of a Hopf bifurcation and determined the conditions for which the bifurcation is either supercritical or subcritical.

González-Olivares and Ramos-Jiliberto [18] studied the following prey-predator model with constant number of prey using refuges as shown in the Figure 1:

$$
\begin{gathered}
\frac{d x}{d t}=\alpha x\left(1-\frac{x}{k}\right)-\frac{\beta(x-m) y}{1+a(x-m)}, \\
\frac{d y}{d t}=-d y+\frac{c \beta(x-m) y}{1+a(x-m)},
\end{gathered}
$$

with $x(0)>m, y(0)>0$, where $x$ and $y$ denote the prey and predator populations, respectively, at any time $t$ and $\alpha$ and $k$, respectively, represent the intrinsic growth rate and carrying capacity of the prey. $m$ is a constant, which represents number of prey which seeks refuge from predation. $d$ is the death rate of the predator and $c$ is the conversion factor. The term $\beta x \mid(1+a x)$ denotes the functional response of the predator, which is termed Holling type II response function (see [2]). They examined the local stability of equilibria and existence of limit cycle. Chen et al. [19] considered the same model and examined also global stability and uniqueness of limit cycle. Also Ji and $\mathrm{Wu}$ [14] considered the prey-predator model with constant prey refuge and a constant-rate prey harvesting $h>0$,

$$
\begin{gathered}
\frac{d x}{d t}=\alpha x\left(1-\frac{x}{k}\right)-\frac{\beta(x-m) y}{1+a(x-m)}-h, \\
\frac{d y}{d t}=-d y+\frac{c \beta(x-m) y}{1+a(x-m)},
\end{gathered}
$$

and studied instability and global stability of the equilibria and uniqueness of limit cycle and showed also the influence of constant prey refuge and constant-rate prey harvesting.

Motivated by the paper of González-Olivares and RamosJiliberto [18], we considered the following prey-predator system:

$$
\begin{gathered}
\frac{d x}{d t}=\alpha x\left(1-\frac{x}{k}\right)-\frac{\beta(x-m) y}{1+a(x-m)}-q_{1} E_{1} x, \\
\frac{d y}{d t}=-d y+\frac{c \beta(x-m) y}{1+a(x-m)}-q_{2} E_{2} y,
\end{gathered}
$$

where $E_{1} \geq 0$ and $E_{2} \geq 0$ denote the harvesting efforts for the prey and predator, respectively. $q_{1} E_{1} x$ and $q_{2} E_{2} y$ represent the catch of the prey and predator population, where $q_{1}$ and $q_{2}$ represent the catchability coefficients, respectively. $\alpha, \beta, k$, $d, a, c, m, q_{1}$, and $q_{2}$ are positive constants.

Our paper is organized in the following way. In Section 2, we have discussed the boundedness of the solutions of system 
(3). All possible equilibria of the system (8) and the stability criterion at those equilibria and uniqueness of limit cycles at interior equilibrium are discussed in Section 3. In Section 4, we have discussed Hopf bifurcation at interior equilibrium. Influence of refuge parameter $m$ and harvesting efforts $E_{1}$ and $E_{2}$ are discussed in Section 5. Numerical simulations are given in Section 6. A brief concluding remark is given in Section 7.

\section{Boundedness of the System}

Boundedness of a model guarantees its validity. The following theorem establishes the uniform boundedness of the model system (3).

Theorem 1. All solutions of the system (3) which start in $R_{+}^{2}$ are uniformly bounded.

Proof. Let $(x(t), y(t))$ be any solution of the system with positive initial conditions $x(0)>m$ and $y(0)>0$.

Now we define the function $W=x+y / c$.

Therefore, time derivative gives

$$
\begin{aligned}
\frac{d W}{d t} & =\frac{d x}{d t}+\frac{1}{c} \frac{d y}{d t} \\
& =\alpha x-\frac{\alpha x^{2}}{k}-q_{1} E_{1} x-\frac{d y}{c}-\frac{q_{2} E_{2} y}{c} .
\end{aligned}
$$

Now for each $v>0$, we have

$$
\begin{aligned}
\frac{d W}{d t}+v W= & \alpha x-\frac{\alpha x^{2}}{k}-q_{1} E_{1} x \\
& +v x+\frac{v y}{c}-\frac{d y}{c}-\frac{q_{2} E_{2} y}{c} .
\end{aligned}
$$

We have $d W / d t+v W \leq(k / 4 \alpha)\left(\alpha+v-q_{1} E_{1}\right)^{2}-(1 / c)(d+$ $\left.q_{2} E_{2}-v\right)$.

Let us choose $v>d+q_{2} E_{2}$; then the right-hand side is positive. As we assume that both $E_{1}$ and $E_{2}$ are bounded, the right-hand side is bounded for all $(x, y) \in R_{+}^{2}$.

Thus we choose a $u>0$ such that $d W / d t+v W<u$.

Applying the theory of differential inequality [20], we obtain

$$
\begin{gathered}
0<W(x, y)<\frac{u}{v}\left(1-e^{-v t}\right)+\frac{W(x(0), y(0))}{e^{v t}}, \\
\text { for } t \rightarrow \infty, \quad 0<W<\frac{u}{v}
\end{gathered}
$$

Thus, all solutions of the system (3) that start in $R_{+}^{2}$ are confined to the region $B$, where

$$
B=\left\{(x, y) \in R_{+}^{2}: W=\frac{u}{v}+\varepsilon \text {, for any } \varepsilon>0\right\} .
$$

This completes the theorem.
Now for simplicity, let us introduce $X=x-m$; then the system (3) of equations changes to (still denote $X=x$ )

$$
\begin{aligned}
\frac{d x}{d t}= & \alpha(x+m)\left(1-\frac{x+m}{k}\right) \\
& -\frac{\beta x y}{1+a x}-q_{1} E_{1}(x+m), \\
\frac{d y}{d t}= & -d y+\frac{c \beta x y}{1+a x}-q_{2} E_{2} y .
\end{aligned}
$$

\section{The Steady States and Their Stability}

We now study the existence and nature of the steady states. Particularly we are interested in the interior equilibrium of the system. To begin with, we list all possible steady states of the system (8) as follows:

(i) equilibrium in the absence of predator $P_{1}\left(x_{1}, 0\right)$, where $x_{1}=\left(k \alpha-m \alpha-q_{1} E_{1} k\right) / \alpha$ exists provided $k \alpha>m \alpha+q_{1} E_{1} k$

(ii) the interior equilibrium $P^{*}\left(x^{*}, y^{*}\right)$, where

$$
\begin{gathered}
x^{*}=\frac{d+q_{2} E_{2}}{\beta c-a d-a q_{2} E_{2}}, \\
y^{*}=\frac{1+a x^{*}}{\beta k x^{*}}\left(\alpha\left(x^{*}+m\right)\left(k-m-x^{*}\right)\right. \\
\left.-q_{1} E_{1} k\left(x^{*}+m\right)\right) .
\end{gathered}
$$

If $\beta c>a d+a q_{2} E_{2}$ holds and any of the following conditions hold:

(H1) $A>0, B>0, A^{2} \geq 4 B$, and $\left(A-\sqrt{A^{2}-4 B}\right) / 2<m<$ $\left(A+\sqrt{A^{2}-4 B}\right) / 2$,

(H2) $A>0, B<0$, and $0<m<\left(A+\sqrt{A^{2}-4 B}\right) / 2$,

(H3) $A<0, B<0$, and $0<m<\left(A+\sqrt{A^{2}-4 B}\right) / 2$,

where $A=k-2 x^{*}-q_{1} E_{1} k / \alpha$ and $B=\left(x^{*}\right)^{2}-k x^{*}+q_{1} E_{1} k x^{*} / \alpha$, then the interior equilibrium $P^{*}\left(x^{*}, y^{*}\right)$ exists.

For the stability analysis of the equilibria, let the variational matrix of the system (8) at an arbitrary point $(x, y)$ be $M(x, y)$.

At $P_{1}\left(x_{1}, 0\right)$, the eigenvalues of the variational matrix $M\left(x_{1}, 0\right)$ are $E_{1} q_{1}-\alpha$ and $c \beta x_{1} /\left(1+a x_{1}\right)-d-q_{2} E_{2}$.

Now if $\left(\alpha / q_{1}\right)\left\{(k-m) / k-\left(d+q_{2} E_{2}\right) / k\left(c \beta-a d-a q_{2} E_{2}\right)\right\}<$ $E_{1}<\alpha / q_{1}$ and $\beta c>a d+a q_{2} E_{2}$, then both eigenvalues are negative and hence $P_{1}\left(x_{1}, 0\right)$ is locally asymptotically stable.

To determine the local stability character of the interior equilibrium $P^{*}\left(x^{*}, y^{*}\right)$, we compute the variational matrix $M\left(x^{*}, y^{*}\right)$ at $\left(x^{*}, y^{*}\right)$.

The characteristic equation of the variational matrix $M\left(x^{*}, y^{*}\right)$ is given by

$$
\gamma^{2}+\gamma\left(\frac{2 m \alpha}{k}-M_{1}\right)+M_{2}=0,
$$


where

$$
\begin{gathered}
M_{1}=\alpha-q_{1} E_{1}-\frac{\beta y^{*}}{(1+a x)^{2}}-\frac{2 x^{*} \alpha}{k}, \\
M_{2}=\frac{c \beta^{2} x^{*} y^{*}}{\left(1+a x^{*}\right)^{3}}>0 .
\end{gathered}
$$

Routh-Hurwitz criterion states that all roots of the characteristic equation (10) have negative real parts if $\left(2 m \alpha / k-M_{1}\right)>$ 0 .

Therefore, interior equilibrium $P^{*}\left(x^{*}, y^{*}\right)$ will be asymptotically stable if $m>M_{1} k / 2 \alpha$, and unstable if $m<M_{1} k / 2 \alpha$.

Theorem 2. If $P^{*}\left(x^{*}, y^{*}\right)$ exists with $m>M_{1} k / 2 \alpha$ then $P^{*}\left(x^{*}, y^{*}\right)$ is locally asymptotically stable.

\subsection{Global Stability for the Interior Equilibrium}

Theorem 3. If $R(x)>0$, then the system (8) will be globally asymptotically stable around the interior equilibrium $P^{*}\left(x^{*}\right.$, $\left.y^{*}\right)$.

Proof. To show the global stability of system (8), we define a Lyapunov function as follows:

$$
\begin{aligned}
V(x, y)= & A_{1}\left(x-x^{*}-x^{*} \ln \frac{x}{x^{*}}\right) \\
& +B_{1}\left(y-y^{*}-y^{*} \ln \frac{y}{y^{*}}\right),
\end{aligned}
$$

where $A_{1}$ and $B_{1}$ are positive constants to be determined in the subsequent steps.

The time derivative along the trajectories of (8) is

$$
\begin{aligned}
\frac{d V}{d t}= & A_{1} \frac{x-x^{*}}{x} \frac{d x}{d t}+B_{1} \frac{y-y^{*}}{y} \frac{d y}{d t} \\
= & A_{1}\left(x-x^{*}\right)\left[\alpha\left(1+\frac{m}{x}\right)\left(1-\frac{x+m}{k}\right)\right. \\
& \left.\quad-\frac{\beta y}{1+a x}-q_{1} E_{1}-\frac{q_{1} E_{1} m}{x}\right] \\
& +B_{1}\left(y-y^{*}\right)\left(-d-q_{2} E_{2}+\frac{c \beta x}{1+a x}\right) \\
= & -A_{1} \frac{\left(x-x^{*}\right)^{2}}{x^{*}}\left[\frac{\alpha x^{*}}{k}+\frac{\alpha m}{x}\left(1-\frac{m}{k}\right)-\frac{q_{1} E_{1} m}{x}\right. \\
& +\beta\left[c B_{1}-A_{1}\left(1+a x^{*}\right)\right] \frac{\left(x-x^{*}\right)\left(y-y^{*}\right)}{(1+a x)\left(1+a x^{*}\right)} \\
= & -A_{1} \frac{\left(x-x^{*}\right)^{*}}{x^{*}} R(x) \\
& +\beta\left[c B_{1}-A_{1}\left(1+a x^{*}\right)\right] \frac{\left(x-x^{*}\right)\left(y-y^{*}\right)}{(1+a x)\left(1+a x^{*}\right)},
\end{aligned}
$$

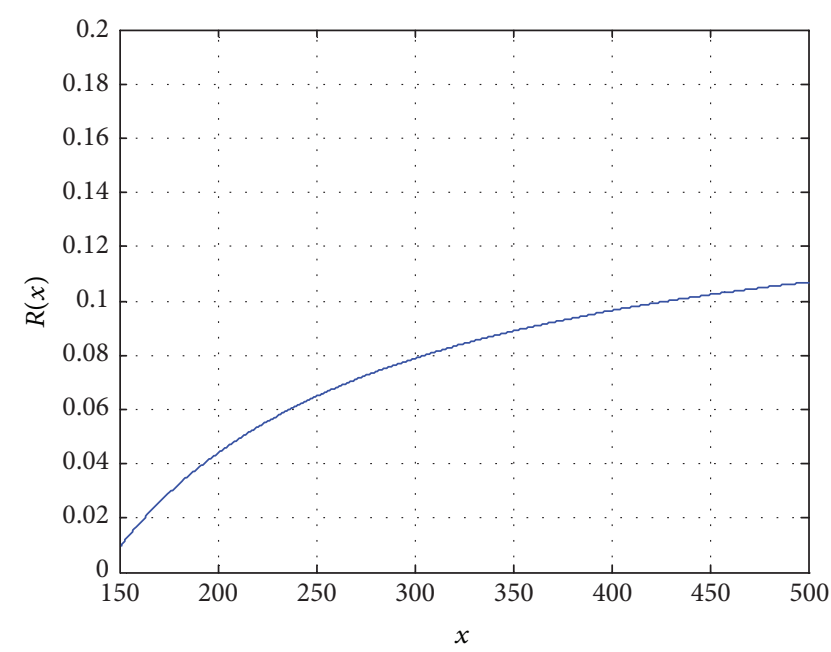

Figure 2: Graph of $R(x)$.

where $R(x)=\left[\alpha x^{*} / k+(\alpha m / x)(1-m / k)-q_{1} E_{1} m / x-\right.$ $\left.a \beta x^{*} y^{*} /(1+a x)\left(1+a x^{*}\right)\right]$.

Choosing $A_{1}=1$ and $B_{1}=\left(1+a x^{*}\right) / c$, we have

$$
\frac{d V}{d t}=-\frac{\left(x-x^{*}\right)^{2}}{x^{*}} R(x) .
$$

Thus if $R(x)>0$, then $d V / d t<0$. This completes the proof.

In Figure 2, we show that $R(x)$ may be positive for some positive value of $x$ by using the numerical value of the parameters $\alpha=8, a=0.5, \beta=4, c=0.8, d=2, k=50$, $q_{1}=0.6, q_{2}=0.2, E_{1}=0.4, E_{2}=0.2$, and $m=0.75$.

3.2. Uniqueness of Limit Cycles. It is known that for preypredator systems, existence and stability of a limit cycle are related to the existence and stability of a positive equilibrium. If the limit cycles do not exist, in this case, the equilibrium is globally asymptotically stable. On the other hand, if the positive equilibrium exists and is unstable, there must occur at least one limit cycle.

Let us consider system (8) in the form

$$
\begin{gathered}
\frac{d x}{d t}=x g(x)-y h(x), \quad x(0)>0, \\
\frac{d y}{d t}=y\left(-d-q_{2} E_{2}+q(x)\right), \quad y(0)>0,
\end{gathered}
$$

where $g(x)=\alpha(1-x / k)-2 \alpha m / k-q_{1} E_{1}+(1 / x)\left(\alpha m-\alpha m^{2} / k-\right.$ $\left.q_{1} E_{1} m\right), h(x)=\beta x /(1+a x)$, and $q(x)=c \beta x /(1+a x)$.

Now we consider the following theorem (see [21]) regarding uniqueness of limit cycles of the previous system.

Theorem 4. Suppose for system (15) that

$$
\frac{d}{d x}\left(\frac{x g^{\prime}(x)+g(x)-x g(x)\left(h^{\prime}(x) / h(x)\right)}{-d-q_{2} E_{2}+q(x)}\right) \leq 0
$$


in $0 \leq x<x^{*}$ and $x^{*}<x \leq k$. Then the previous system has exactly one limit cycle which is globally asymptotically stable with respect to the set

$$
\{(x, y): x>0, y>0\} \backslash\left\{P^{*}\left(x^{*}, y^{*}\right)\right\} .
$$

Following Theorem 4, we may state that when $m<M_{1} k / 2 \alpha$, system (8) has unique globally stable limit cycle. Thus we see that when the system is unstable, there exists a unique globally stable limit cycle.

\section{Hopf Bifurcation}

To discuss Hopf bifurcation of the system (8) we take the help of the paper [22].

Let us now consider system (8) in the form

$$
\begin{gathered}
\frac{d x}{d t}=h(x)(f(x)-y), \\
\frac{d y}{d t}=c y\left(h(x)-d^{\prime}\right),
\end{gathered}
$$

where $f(x)=(\alpha / k \beta)(k-x)(1+a x)-(2 \alpha m / k \beta)(1+$ $a x)-\left(q_{1} E_{1} / \beta\right)(1+a x)+(1 / x \beta)\left(\alpha m-\alpha m^{2} / k-q_{1} E_{1} m\right)(1+$ $a x), h(x)=\beta x /(1+a x)$, and $d^{\prime}=\left(d+q_{2} E_{2}\right) / c$.

Let $\left(\delta, y_{\delta}\right)$ be the interior equilibrium of the system (18), where

$$
\begin{gathered}
\delta=\frac{d+q_{2} E_{2}}{\beta c-a d-a q_{2} E_{2}}, \\
y_{\delta}=\frac{1+a \delta}{\beta k \delta}\left(\alpha(\delta+m)(k-m-\delta)-q_{1} E_{1} k(\delta+m)\right) .
\end{gathered}
$$

In this section, we examined the Hopf bifurcation occurring at $\left(\bar{\delta}, y_{\bar{\delta}}\right)$ taking $\delta$ as a bifurcation parameter.

Variational matrix of the system $(18)$ at $\left(\delta, y_{\delta}\right)$ is

$$
J=\left(\begin{array}{cc}
W(\delta) & Y(\delta) \\
Z(\delta) & 0
\end{array}\right),
$$

where $W(\delta)=h(\delta) f^{\prime}(\delta), Y(\delta)=-h(\delta)$, and $Z(\delta)=$ $c f(\delta) h^{\prime}(\delta)$.

The characteristic equation of the variational matrix $J$ is given by $\mu^{2}-\mu T+D=0$, where $T=\operatorname{tr} J=W(\delta)$ and $D=$ $\operatorname{det} J=-Y(\delta) Z(\delta)$.

Now if $T<0$ and $D>0$, then $\left(\delta, y_{\delta}\right)$ is locally asymptotically stable. On the other hand, if $T>0$ and $D>0$ then $\left(\delta, y_{\delta}\right)$, is unstable. Also $T(\bar{\delta})=0$ and the Jacobian matrix $J(\bar{\delta})$ has a pair of imaginary eigenvalues $\mu= \pm i \sqrt{-Y(\bar{\delta}) Z(\bar{\delta})}$. Let $\mu=\beta(\delta) \pm i \omega(\delta)$ be the roots of $\mu^{2}-\mu T+D=0$ when $\delta$ is near $\bar{\delta}$; then

$$
\begin{aligned}
& \beta(\delta)=\frac{A(\delta)}{2}, \quad \omega(\delta)=\frac{\sqrt{-4 Y(\delta) Z(\delta)-\{W(\delta)\}^{2}}}{2}, \\
& \beta^{\prime}(\delta)=\frac{\left\{h(\delta) f^{\prime}(\delta)\right\}^{\prime}}{2}=\frac{\left\{h^{\prime}(\delta) f^{\prime}(\delta)+h(\delta) f^{\prime \prime}(\delta)\right\}}{2} .
\end{aligned}
$$

$\bar{\delta}$ is a root of $f^{\prime}(\delta)=0\left(\right.$ as $\left.h(\delta)=d^{\prime} \neq 0\right)$; that is $\delta=\bar{\delta}$, satisfies the equation

$$
\begin{gathered}
2 \alpha a \delta^{3}+\left(2 \alpha a m+q_{1} E_{1} a k+\alpha-a \alpha k\right) \delta^{2} \\
+\left(\alpha m k-\alpha m^{2}-q_{1} E_{1} k m\right)=0,
\end{gathered}
$$

and also $f^{\prime \prime}(\bar{\delta}) \neq 0$.

Therefore, $\left.\beta^{\prime}(\delta)\right|_{\delta=\bar{\delta}}=h(\bar{\delta}) f^{\prime \prime}(\bar{\delta}) / 2 \neq 0$ as $h(\bar{\delta}) \neq 0$.

The system (18) undergoes a Hopf bifurcation at $\left(\bar{\delta}, y_{\bar{\delta}}\right)$ when $\delta=\bar{\delta}$. By further analysis, we determined that the bifurcation is either supercritical or subcritical by the first Lyapunov coefficient (see [23-25])

$$
\begin{aligned}
a(\bar{\delta})=\frac{1}{16 h^{\prime}(\bar{\delta})}[ & f^{\prime \prime \prime}(\bar{\delta}) h(\bar{\delta}) h^{\prime}(\bar{\delta})+2\left\{h^{\prime}(\bar{\delta})\right\}^{2} f^{\prime \prime}(\bar{\delta}) \\
& \left.-f^{\prime \prime}(\bar{\delta}) h^{\prime \prime}(\bar{\delta}) h(\bar{\delta})\right] .
\end{aligned}
$$

The computation of $a(\bar{\delta})$ is technical, and the detailed calculations are given in the appendix and only the results are stated in the following theorem.

Theorem 5. The system (18) undergoes a Hopf bifurcation at $\left(\bar{\delta}, y_{\bar{\delta}}\right)$; the Hopf bifurcation is supercritical and backward (subcritical and forward, resp.) if $a(\bar{\delta})<0(a(\bar{\delta})>0)$, where $a(\bar{\delta})$ is defined in (23).

\section{Influence of the Parameter $m$ and Harvesting Efforts $E_{1}$ and $E_{2}$}

Interior equilibrium of the system (8) is $P^{*}\left(x^{*}, y^{*}\right)$, where

$$
\begin{gathered}
x^{*}=\frac{d+q_{2} E_{2}}{\beta c-a d-a q_{2} E_{2}}, \\
y^{*}=\frac{1+a x^{*}}{\beta k x^{*}}\left(\alpha\left(x^{*}+m\right)\left(k-m-x^{*}\right)\right. \\
\left.-q_{1} E_{1} k\left(x^{*}+m\right)\right) .
\end{gathered}
$$

Notice that the scaling from system (3) to system (8) is $X=$ $x-m$. Let the interior equilibrium of the system (3) be $P^{\prime \prime}\left(x^{\prime \prime}, y^{\prime \prime}\right)$, where

$$
\begin{gathered}
x^{\prime \prime}=\frac{d+q_{2} E_{2}}{\beta c-a d-a q_{2} E_{2}}+m, \\
y^{\prime \prime}=\frac{1+a\left(x^{\prime \prime}-m\right)}{\beta k\left(x^{\prime \prime}-m\right)}\left(\alpha x^{\prime \prime}\left(k-x^{\prime \prime}\right)-q_{1} E_{1} k x^{\prime \prime}\right) .
\end{gathered}
$$

The model (3) is a one-prey-one-predator model with constant prey refuge. Now we discuss the influence of the prey refuge on the model dynamics.

Now $d x^{\prime \prime} / d m=1>0$.

Therefore, $x^{\prime \prime}$ is a strictly increasing function of $m$. 
TABLE 1

\begin{tabular}{lcc}
\hline$m$ & $x^{\prime \prime}$ & $y^{\prime \prime}$ \\
\hline 0.1 & 1.03578 & 3.0847 \\
0.2 & 1.13578 & 3.37539 \\
0.398969 & 1.33578 & 3.95004 \\
0.6 & 1.53578 & 4.5256 \\
0.75 & 1.68578 & 4.95175 \\
1 & 1.93578 & 5.65572 \\
3 & 3.93578 & 11.0052 \\
10 & 10.93578 & 25.7753 \\
20 & 20.93578 & 36.2088 \\
$23.31422^{*}$ & 24.25002 & 36.8980 \\
24 & 24.93578 & 36.8685 \\
25 & 25.93578 & 36.7197 \\
30 & 30.93578 & 34.0934 \\
35 & 35.93578 & 28.3297 \\
40 & 40.93578 & 19.4288 \\
47 & 47.93578 & 7.39071 \\
47.5 & 48.43578 & 0.195172 \\
\hline
\end{tabular}

${ }^{*}$ At $m=23.31422$, the value of $y^{\prime \prime}$ is maximum.

That is, increasing the amount of prey refuge can increase prey population.

To check the effect of $m$ on predator, differentiating $y^{\prime \prime}$ with respect to $m$, we get $d y^{\prime \prime} / d m=-\left(2 \alpha\left(1+a x^{*}\right) / \beta x^{*} k\right)(m+$ $\left.x^{*}-k / 2+q_{1} E_{1} k / 2 \alpha\right)$, where $x^{*}=\left(d+q_{2} E_{2}\right) /\left(\beta c-a d-a q_{2} E_{2}\right)$.

Case 1. If $m>k / 2-x^{*}-q_{1} E_{1} k / 2 \alpha$, then $y^{\prime \prime}$ is a monotonically decreasing function of $m$.

That is, if the value of prey refuge $m$ gradually increases above threshold value $k / 2-x^{*}-q_{1} E_{1} k / 2 \alpha$, then the predator population gradually decreases.

Case 2. If $m<k / 2-x^{*}-q_{1} E_{1} k / 2 \alpha$, then $y^{\prime \prime}$ is a monotonically increasing function of $m$.

That is, if the value of prey refuge $m$ gradually decreases below threshold value $k / 2-x^{*}-q_{1} E_{1} k / 2 \alpha$, then the predator population gradually increases.

Case 3. If $=k / 2-x^{*}-q_{1} E_{1} k / 2 \alpha$, then $y^{\prime \prime}$ has a maximum value.

To construct Table 1 and Figure 3, we take $\alpha=8, a=0.5$, $\beta=4, c=0.8, d=2, k=50, q_{1}=0.6, q_{2}=0.2$, $E_{1}=0.4$, and $E_{2}=0.2$ in appropriate units. From the analysis shown we see that increasing the amount of prey refuge can increase prey population and that increasing the amount of prey refuge can increase the density of predator species, and this happened due to predator species still having enough food for predation with $m$ being small, but if the prey refuge is larger than a threshold, that is, as the prey refuge becomes large enough, then the increasing amount of prey refuge can decrease predator species, and this happened due to the loss of food for predator species.

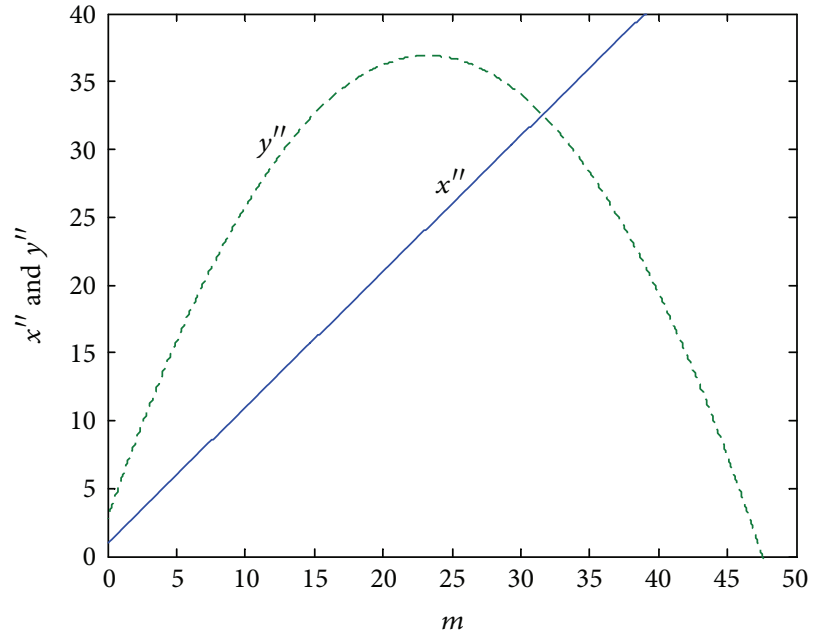

FIgURE 3: Change of $x^{\prime \prime}$ and $y^{\prime \prime}$ with the change of the refuge parameter $m$.

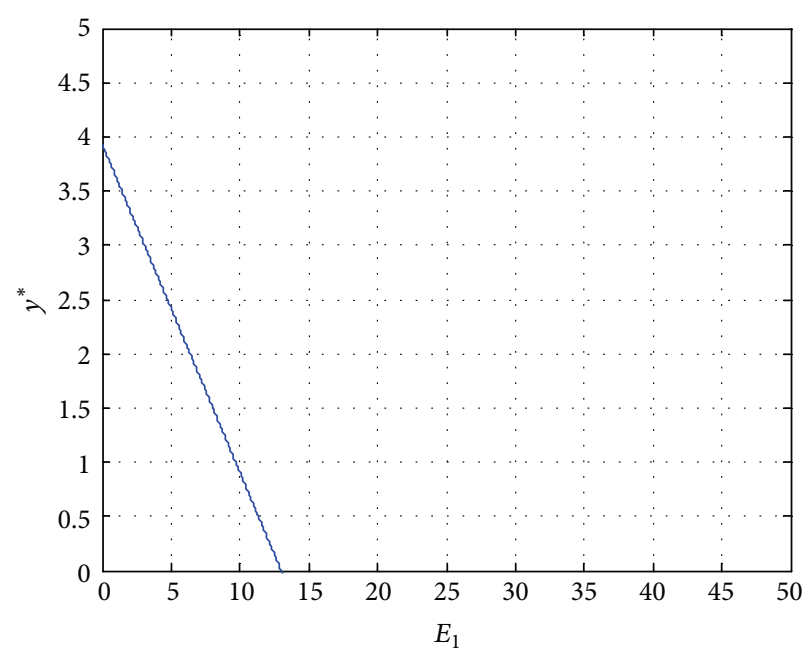

FIGURE 4: Change of $y^{*}$ with the change of the harvesting effort $E_{1}$.

Influence of the Harvesting Efforts $E_{1}$ and $E_{2}$. Interior equilibrium of the system $(8)$ is $P^{*}\left(x^{*}, y^{*}\right)$, where

$$
\begin{gathered}
x^{*}=\frac{d+q_{2} E_{2}}{\beta c-a d-a q_{2} E_{2}}, \\
y^{*}=\frac{1+a x^{*}}{\beta k x^{*}}\left(\alpha\left(x^{*}+m\right)\left(k-m-x^{*}\right)-q_{1} E_{1} k\left(x^{*}+m\right)\right) .
\end{gathered}
$$

At first let $m$ and $E_{2}$ be fixed.

It is observed that $x^{*}$ is independent of the parameter $E_{1}$, whereas the value of $y^{*}$ depends on $E_{1}$. Therefore $E_{1}$ has no effect on the interior equilibrium level on $x$.

To check the effect of $E_{1}$ on predator, differentiating $y^{*}$ with respect to $E_{1}$, we get

$$
\frac{d y^{*}}{d E_{1}}=-\frac{q_{1}\left(1+a x^{*}\right)\left(m+x^{*}\right)}{\beta x^{*}}<0 .
$$

This shows that $y^{*}$ is a strictly decreasing function of $E_{1}$. 
TABLE 2

\begin{tabular}{lcc}
\hline$E_{1}$ & $x^{*}$ & $y^{*}$ \\
\hline 1 & 0.93578 & 3.62755 \\
3 & 0.93578 & 3.02248 \\
5 & 0.93578 & 2.4174 \\
6 & 0.93578 & 2.11487 \\
7.62878 & 0.93578 & 1.6221 \\
9.6 & 0.93578 & 1.02574 \\
11 & 0.93578 & 0.602186 \\
12 & 0.93578 & 0.29965 \\
12.5 & 0.93578 & 0.148382 \\
12.9 & 0.93578 & 0.0273671 \\
\hline
\end{tabular}

TABLE 3

\begin{tabular}{lcc}
\hline$E_{2}$ & $x^{*}$ & $y^{*}$ \\
\hline 1 & 1.04762 & 3.79968 \\
5 & 1.76471 & 4.15139 \\
10 & 3.33333 & 5.23819 \\
15 & 7.14286 & 7.79394 \\
17 & 10.8 & 9.77236 \\
19.4068 & 22.6799 & 12.578 \\
19.6 & 24.6667 & 12.4993 \\
20 & 30.00 & 11.5087 \\
20.5 & 40.6667 & 6.11798 \\
20.7 & 47.2308 & 0.539829 \\
\hline
\end{tabular}

To construct Table 2 and Figure 4, we take $\alpha=8, a=0.5$, $\beta=4, c=0.8, d=2, k=50, q_{1}=0.6, q_{2}=0.2, m=$ 0.35 , and $E_{2}=0.2$ in appropriate units. From the analysis shown we see that as the harvesting effort $E_{1}$ increases, the prey species remain unchanged but predator species decrease and this happens due to loss of food for predator species and goes to extinction when $E_{1}$ is large.

Now let $m$ and $E_{1}$ be fixed; then we have

$$
\frac{d x^{*}}{d E_{2}}=\frac{\beta c q_{2}}{\left(\beta c-a d-a E_{2} q_{2}\right)^{2}}>0
$$

Therefore $x^{*}$ is a strictly increasing function of $E_{2}$.

Also

$$
\begin{array}{r}
\frac{d y^{*}}{d E_{2}}=\left[\frac{\alpha m^{2}}{\left(x^{*}\right)^{2}}+\frac{q_{1} E_{1} k m}{\left(x^{*}\right)^{2}}+2 a m \alpha+a k \alpha-\alpha\right. \\
\left.-2 \alpha a x^{*}-q_{1} E_{1} k a-\frac{k \alpha m}{\left(x^{*}\right)^{2}}\right] \frac{d x^{*}}{d E_{2}} .
\end{array}
$$

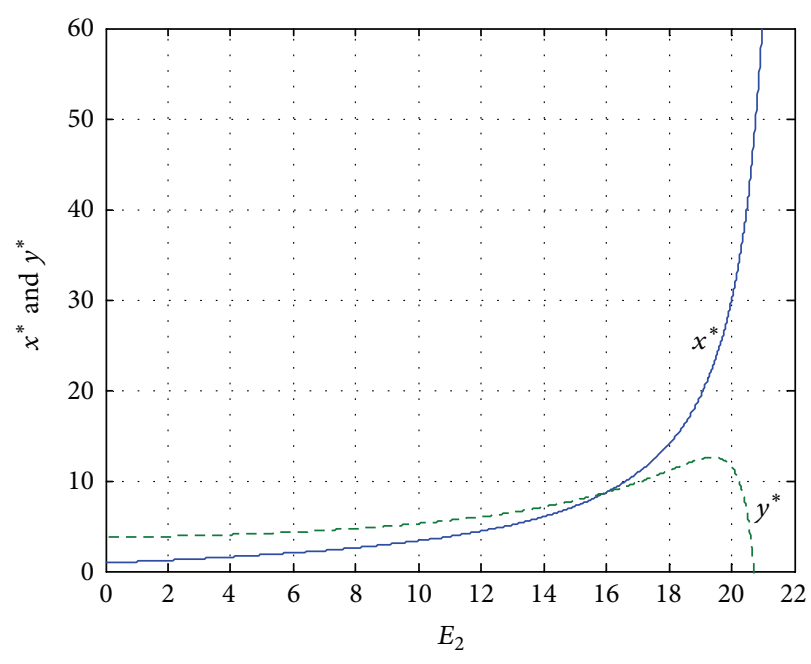

FIGURE 5: Variation of $x^{*}$ and $y^{*}$ with the change of the harvesting effort $E_{2}$.

Since $d x^{*} / d E_{2}>0$, therefore

$$
\begin{array}{r}
\frac{d y^{*}}{d E_{2}}>0 \quad \text { if } \frac{\alpha m^{2}}{\left(x^{*}\right)^{2}}+\frac{q_{1} E_{1} k m}{\left(x^{*}\right)^{2}}+2 a m \alpha+a k \alpha \\
>\alpha+2 a \alpha x^{*}+q_{1} E_{1} k a+\frac{k \alpha m}{\left(x^{*}\right)^{2}}, \\
\frac{d y^{*}}{d E_{2}}<0 \quad \text { if } \frac{\alpha m^{2}}{\left(x^{*}\right)^{2}}+\frac{q_{1} E_{1} k m}{\left(x^{*}\right)^{2}}+2 a m \alpha+a k \alpha \\
<\alpha+2 a \alpha x^{*}+q_{1} E_{1} k a+\frac{k \alpha m}{\left(x^{*}\right)^{2}} .
\end{array}
$$

Thus as the harvesting effort $E_{2}$ increases, the predator population increases when $E_{2}$ is smaller than the threshold value. But if the harvesting effort $E_{2}$ gradually increases above the threshold value, that is, as the harvesting effort $E_{2}$ becomes large enough, then the increasing amount of the harvesting effort $E_{2}$ can decrease predator population.

To construct Table 3 and Figure 5, we take $\alpha=8, a=$ $0.5, \beta=4, c=0.8, d=2, k=50, q_{1}=0.6, q_{2}=$ $0.2, E_{1}=0.5$, and $m=0.35$ in appropriate units. From the analysis shown we see that as the harvesting effort $E_{2}$ increases, the prey and predator species increase. But if the harvesting effort $E_{2}$ increases larger than a threshold value, that is, as the harvesting effort $E_{2}$ becomes large enough, then increasing amount of the harvesting effort $E_{2}$ can increase the prey species but will decrease predator species and go to extinction of the predator species when $E_{2}$ is large.

\section{Numerical Simulation}

As the problem is not a case study, the real-world data are not available for this model. We, therefore, take here some hypothetical data with the sole purpose of illustrating the results that we have established in the previous sections. Let us consider the parameters of the system as $\alpha=8, a=0.5$, 


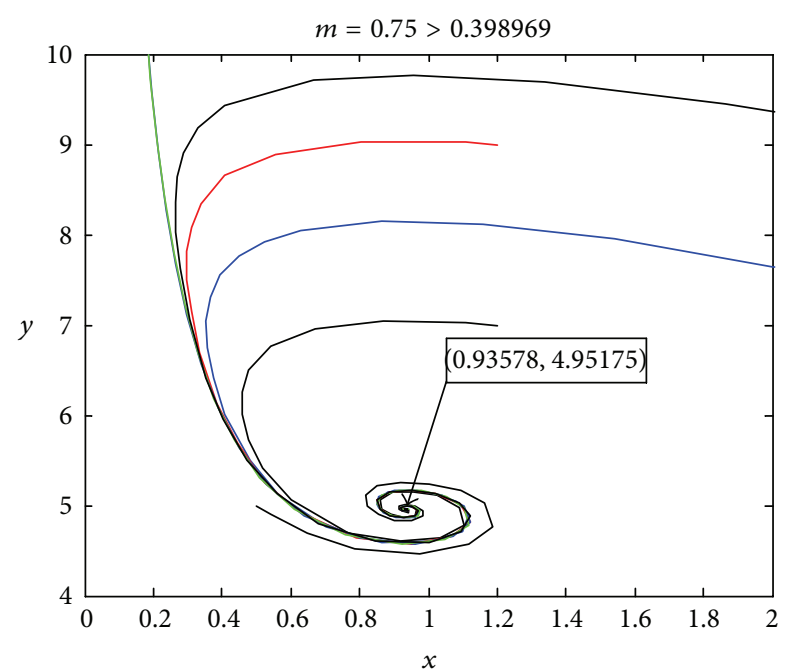

FIGURE 6: Phase space trajectories corresponding to different initial levels, which shows that $(0.93578,4.95175)$ is a global attractor.

$\beta=4, c=0.8, d=2, k=50, q_{1}=0.6, q_{2}=0.2, E_{1}=0.4$, and $E_{2}=0.2$ in appropriate units. For these value of parameters, we get the critical value of $m$ as $m^{*}=0.398969$. Thus it is easy to verify that for this set of parameters, the system (8) is locally asymptotically stable around its interior equilibrium $P^{*}\left(x^{*}, y^{*}\right)$ for $m>m^{*}$ and is unstable for $m<$ $m^{*}$. Thus for $m=m^{*}=0.398969$ the system (8) undergoes a Hopf bifurcation. Now for $m=0.75$, we have interior equilibrium $(0.93578,4.95175)$ which is asymptotically stable (see Figure 6), but $m=0.2$, and the interior equilibrium $(0.93578,3.37539)$ is unstable (see Figure 7$)$. Thus taking $m$ as a control parameter, it is possible to drive the preypredator system to require equilibrium and to prevent the cycle behaviour of the system. From Figures 8, 9, 10, and 11, we see that $E_{1}$ and $E_{2}$ may also be used as controls for the system (8); Hopf bifurcation occurs when $E_{1}=E_{1}^{*}=7.62878$ (here $E_{2}=0.2, m=0.35$ ) and $E_{2}=E_{2}^{*}=19.4068$ (here $E_{1}=0.5$, $m=0.35$ ). For the previous values of parameters and $m=$ 0.398969 , we obtained one value of $\delta$ say $\bar{\delta}=0.93578$ and $a(\bar{\delta})=-0.332712<0$. Thus we may conclude that the Hopf bifurcation around the interior equilibrium is supercritical and backward.

\section{Concluding Remarks}

This paper deals with a prey-predator model with Holling type II functional response incorporating a constant prey refuge and independent harvesting in either species. Oscillatory behavior and existence of limit cycles in harvested prey-predator system are common in nature. It is noted that constant prey refuge plays an important role in the dynamics of the proposed model system. It is also observed from the obtained results that constant prey refuge can cause an unstable equilibrium to become stable, and even a simple Hopf bifurcation occurred when the parameter $m$ passes through its critical value. There exists a threshold

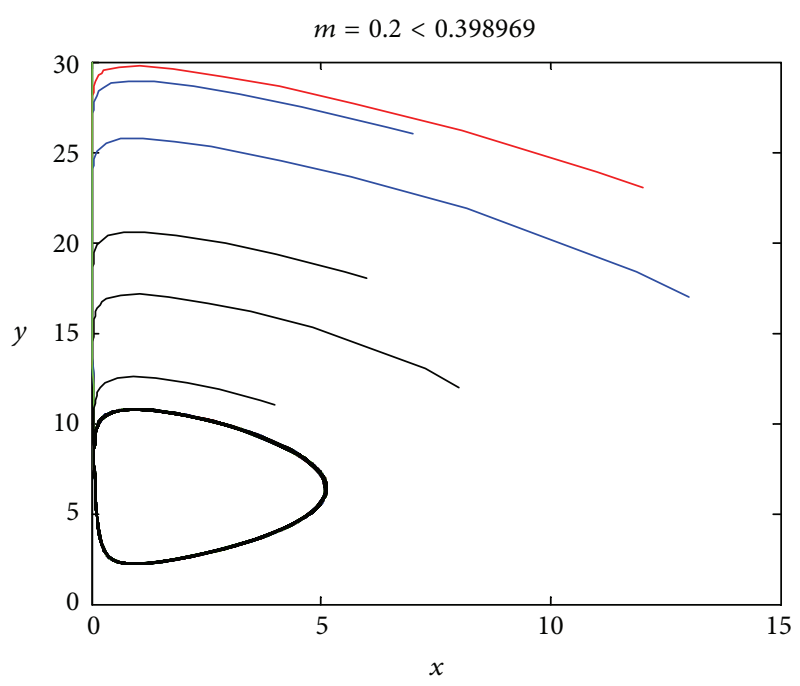

FIGURE 7: There is a stable limit cycle surrounding $(0.93578,3.3754)$ with $m=0.2$.

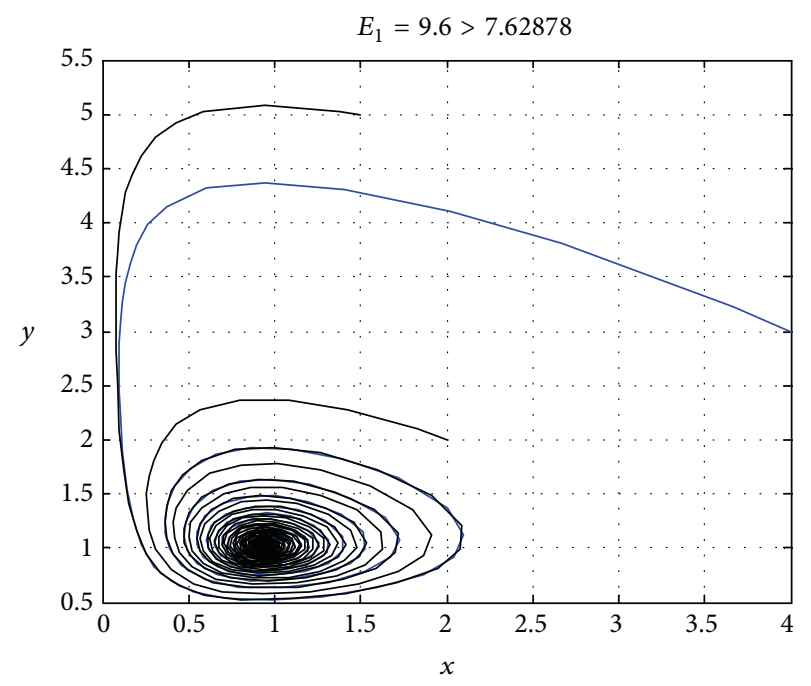

FIGURE 8: Phase space trajectories corresponding to different initial levels. Here $E_{1}=9.6, E_{2}=0.2$, and $m=0.35$.

value of $m$, such that for the prey refuge smaller than this threshold, increasing the amount of prey refuge can increase the predator population, and if the prey refuge is larger than the threshold, increasing the amount of prey refuge can decrease the predator population. We have proved that exactly one stable limit cycle occurs when the positive equilibrium is unstable. We also determined the critical value of $\delta$ at which Hopf bifurcation occurs and observed that the bifurcation is supercritical and backward. It was also found that it is possible to control the system in such a way that the system approaches a required state, using the efforts $E_{1}$ and $E_{2}$ as controls.

Our analytical results and numerical simulation also indicate that dynamic behavior of the model not only depends on the prey refuge parameter $m$ but also depends on harvesting efforts $E_{1}$ and $E_{2}$. Hence it is possible to control the system in 


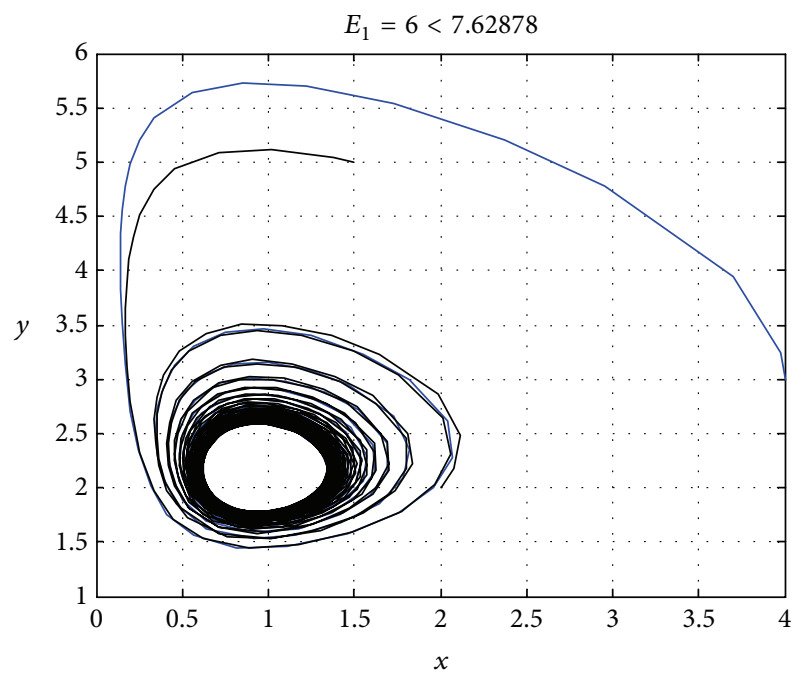

FIgURE 9: There is a stable limit cycle surrounding (0.93578, 2.11487) with $E_{1}=6, E_{2}=0.2$, and $m=0.35$.

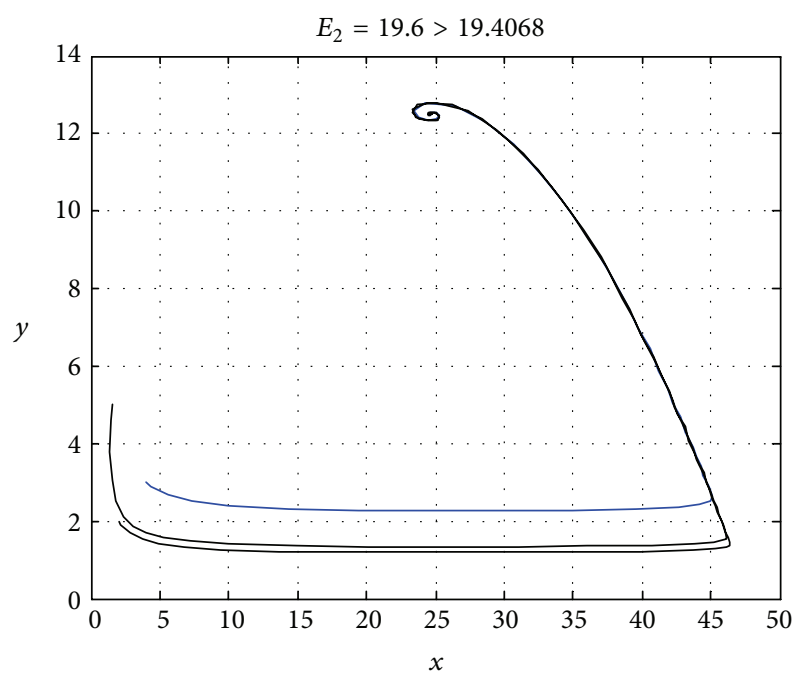

FIGURE 10: Phase space trajectories corresponding to different initial levels with $E_{1}=0.5, E_{2}=19.6$, and $m=0.35$.

such a way that the system approaches a required state, using the harvesting efforts $E_{1}$ and $E_{2}$ or prey refuge $m$ as controls.

In our model, we have considered the catch-rate function based on catch-per-unit-effort hypothesis; that is, $h=q E u(u$ and $E$ denote the prey or predator population and harvesting effort, resp.). But this type of catch-rate function embodies some defects in that (i) it assumes random search for fish, (ii) it assumes equal likelihood of being captured for every fish, (iii) there is unbounded linear increase of $h$ with respect to $E$ for fixed $u$, and (iv) there is unbounded linear increase $h$ with respect to $u$ for a fixed $E$. These unrealistic features can largely be removed by adopting the alternative functional form $h=$ $q E u /\left(n_{1} E+n_{2} u\right)$, where $n_{1}$ and $n_{2}$ are positive constants, but we leave it for our future research work. The entire study of the paper is mainly based on the deterministic framework. On the other hand, it will be more realistic if it is possible

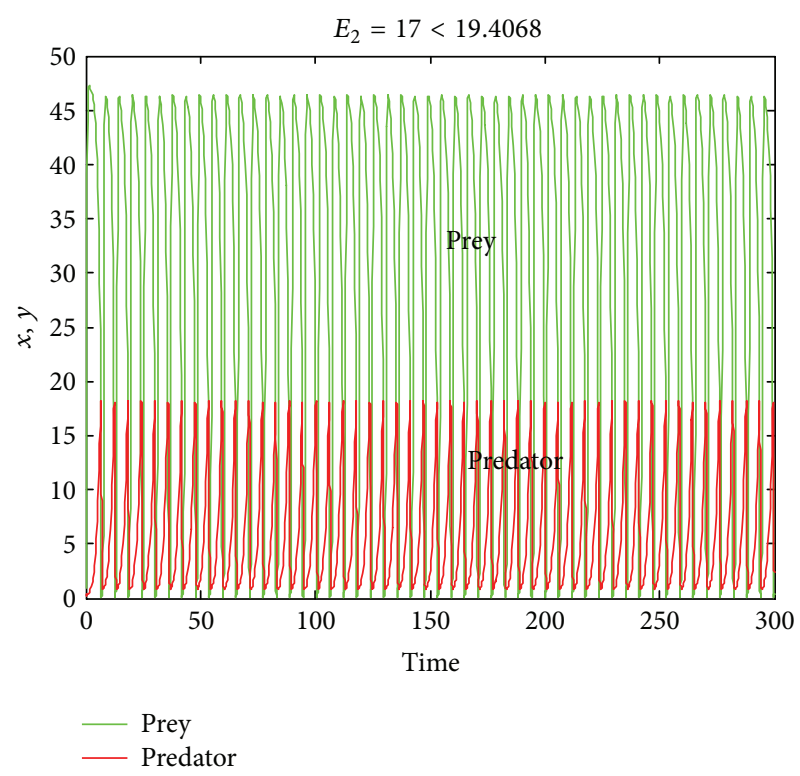

FIgURE 11: There exist Hopf-bifurcating small amplitude periodic solutions with $E_{1}=0.5, E_{2}=17$, and $m=0.35$.

to consider the model system in the stochastic environment due to some ecological fluctuations and other factors. Thus, a future research problem would be considered in stochastic environment.

\section{Appendix}

\section{Detailed Calculation of Formula (23)}

First we translate the equilibrium $\left(\delta, y_{\delta}\right)$ to the origin by translation $\bar{x}=x-\delta, \bar{y}=y-y_{\delta}$. (Still denote $\bar{x}$ and $\bar{y}$ by $x$ and $y$, resp.). Thus the system (18) becomes

$$
\begin{gathered}
\frac{d x}{d t}=h(x+\delta)\left(f(x+\delta)-\left(y+y_{\delta}\right)\right), \\
\frac{d y}{d t}=c\left(y+y_{\delta}\right)\left(h(x+\delta)-d^{\prime}\right) .
\end{gathered}
$$

We write the system (A.1) as follows:

$$
\begin{gathered}
\frac{d x}{d t}=h(x+\delta) f(x+\delta)-h(\delta) f(\delta) \\
-\left(h(x+\delta)\left(y+y_{\delta}\right)-h(\delta) y_{\delta}\right), \\
\frac{d y}{d t}=-c d^{\prime} y+\left(\operatorname{ch}(x+\delta)\left(y+y_{\delta}\right)-\operatorname{ch}(\delta) y_{\delta}\right),
\end{gathered}
$$

where $f(\delta)=y_{\delta}$ and $h(\delta)=d^{\prime}$. 
Now compute the Taylor expansion of related functions

$$
\begin{aligned}
\left(y+y_{\delta}\right) & h(x+\delta)-h(\delta) y_{\delta} \\
= & a_{10} x+a_{01} y+a_{20} x^{2}+a_{11} x y+a_{30} x^{3} \\
& +a_{21} x^{2} y+O\left(|x|^{4},|x|^{3}|y|\right), \\
h(x+ & \delta) f(x+\delta)-h(\delta) f(\delta) \\
= & b_{10} x+b_{20} x^{2}+b_{30} x^{3}+O\left(|x|^{4}\right),
\end{aligned}
$$

where

$$
\begin{gathered}
a_{10}=h^{\prime}(\delta) y_{\delta}, \quad a_{20}=\frac{1}{2} h^{\prime \prime}(\delta) y_{\delta}, \quad a_{30}=\frac{1}{6} h^{\prime \prime \prime}(\delta) y_{\delta}, \\
a_{01}=h(\delta), \quad a_{11}=h^{\prime}(\delta), \quad a_{21}=\frac{1}{2} h^{\prime \prime}(\delta), \\
b_{10}=(f h)^{\prime}(\delta), \quad b_{20}=\frac{1}{2}(f h)^{\prime \prime}(\delta), \quad b_{30}=\frac{1}{6}(f h)^{\prime \prime \prime}(\delta) .
\end{gathered}
$$

Then the system (A.1) becomes

$$
\left(\begin{array}{l}
\frac{d x}{d t} \\
\frac{d y}{d t}
\end{array}\right)=J\left(\begin{array}{l}
x \\
y
\end{array}\right)+\left(\begin{array}{l}
F_{1}(x, y, \delta) \\
F_{2}(x, y, \delta)
\end{array}\right),
$$

where

$$
\begin{gathered}
J=\left(\begin{array}{cc}
W(\delta) & Y(\delta) \\
Z(\delta) & 0
\end{array}\right), \\
F_{1}(x, y, \delta)=\left(b_{20}-a_{20}\right) x^{2}-a_{11} x y+\left(b_{30}-a_{30}\right) x^{3} \\
-a_{21} x^{2} y+O\left(|x|^{4},|x|^{3}|y|\right), \\
F_{2}(x, y, \delta)=c\left(a_{20} x^{2}+a_{11} x y+a_{30} x^{3}\right. \\
\left.+a_{21} x^{2} y+O\left(|x|^{4},|x|^{3}|y|\right)\right) .
\end{gathered}
$$

Now we define a matrix

$$
P=\left(\begin{array}{cc}
1 & 0 \\
N & M
\end{array}\right)
$$

where $N=-W(\delta) / 2 Y(\delta)$ and $M=$ $-\sqrt{-4 Y(\delta) Z(\delta)-W^{2}(\delta)} / 2 Y(\delta)$; then

$$
P^{-1}=\left(\begin{array}{cc}
1 & 0 \\
-\frac{N}{M} & \frac{1}{M}
\end{array}\right) .
$$

By using the linear transformation

$$
\left(\begin{array}{l}
x \\
y
\end{array}\right)=P\left(\begin{array}{l}
u \\
v
\end{array}\right)
$$

we have

$$
\left(\begin{array}{l}
x \\
y
\end{array}\right)=\left(\begin{array}{c}
u \\
N u+M v
\end{array}\right), \quad P^{-1} J P\left(\begin{array}{l}
u \\
v
\end{array}\right)=J(\delta)\left(\begin{array}{l}
u \\
v
\end{array}\right),
$$

where

$$
J(\delta)=\left(\begin{array}{cc}
\beta(\delta) & -\omega(\delta) \\
\omega(\delta) & \beta(\delta)
\end{array}\right) .
$$

Then system (A.5) becomes

$$
\left(\begin{array}{c}
\frac{d u}{d t} \\
\frac{d v}{d t}
\end{array}\right)=J(\delta)\left(\begin{array}{l}
u \\
v
\end{array}\right)+\left(\begin{array}{l}
F^{1}(u, v, \delta) \\
F^{2}(u, v, \delta)
\end{array}\right),
$$

where $\beta(\delta)$ and $\omega(\delta)$ are defined in (21), and

$$
\begin{aligned}
F^{1}(u, v, \delta)= & F_{1}(u, N u+M v, \delta) \\
= & A_{20} u^{2}+A_{11} u v+A_{30} u^{3} \\
& +A_{21} u^{2} v+O\left(|u|^{4},|u|^{3}|v|\right), \\
F^{2}(u, v, \delta)= & -\frac{N}{M} F_{1}(u, N u+M v, \delta) \\
& +\frac{1}{M} F_{2}(u, N u+M v, \delta) \\
= & B_{20} u^{2}+B_{11} u v+B_{30} u^{3}+B_{21} u^{2} v \\
& +O\left(|u|^{4},|u|^{3}|v|\right),
\end{aligned}
$$

where

$$
\begin{gathered}
A_{20}=\left(b_{20}-a_{20}\right)-a_{11} N, \quad A_{11}=-a_{11} M, \\
A_{30}=\left(b_{30}-a_{30}\right)-a_{21} N, \quad A_{21}=-a_{21} M, \\
B_{20}=\frac{c}{M}\left(a_{20}+a_{11} N\right)-\frac{N}{M}\left(b_{20}-a_{20}-a_{11} N\right), \\
B_{11}=c a_{11}+a_{11} N, \\
B_{30}=\frac{c}{M}\left(a_{30}+a_{21} N\right)-\frac{N}{M}\left(b_{30}-a_{30}-a_{21} N\right), \\
B_{21}=c a_{21}+a_{21} N .
\end{gathered}
$$

Rewrite the system (A.12) in a polar coordinate form as:

$$
\begin{gathered}
\dot{r}=\beta(\delta) r+a(\delta) r^{3}+\cdots, \\
\dot{\theta}=\omega(\delta)+c(\delta) r^{2}+\cdots
\end{gathered}
$$

Then the Taylor expansion of (A.15) at $\delta=\bar{\delta}$ yields

$$
\begin{aligned}
\dot{r}= & \beta^{\prime}(\bar{\delta})(\delta-\bar{\delta}) r+a(\bar{\delta}) r^{3} \\
& +O\left(r|\delta-\bar{\delta}|^{2}, r^{3}|\delta-\bar{\delta}|, r^{5}\right), \\
\dot{\theta}= & \omega(\bar{\delta})+\omega^{\prime}(\bar{\delta})(\delta-\bar{\delta})+c(\bar{\delta}) r^{2} \\
& +O\left(|\delta-\bar{\delta}|^{2}, r^{2}|\delta-\bar{\delta}|, r^{4}\right) .
\end{aligned}
$$


In order to determine the stability of the periodic solution, we need to calculate the sign of the coefficient $a(\bar{\delta})$, which is given by

$$
\begin{gathered}
a(\bar{\delta})=\frac{1}{16}\left[F_{u u u}^{1}+F_{u v v}^{1}+F_{u u v}^{2}+F_{v v v}^{2}\right] \\
+\frac{1}{16 \omega(\bar{\delta})}\left[F_{u v}^{1}\left(F_{u u}^{1}+F_{v v}^{1}\right)-F_{u v}^{2}\left(F_{u u}^{2}+F_{v v}^{2}\right)\right. \\
\left.-F_{u u}^{1} F_{u u}^{2}+F_{v v}^{1} F_{v v}^{2}\right],
\end{gathered}
$$

where all partial derivatives are evaluated at the bifurcation point; that is, $(u, v, \delta)=(0,0, \bar{\delta})$. that

Since $v$ is linear in both $F^{1}(u, v, \delta)$ and $F^{2}(u, v, \delta)$, we have

$$
F_{u v v}^{1}=F_{v v v}^{2}=F_{v v}^{1}=F_{v v}^{2}=0,
$$

when $\delta=\bar{\delta}, N_{\bar{\delta}}=\left.N\right|_{\delta=\bar{\delta}}=0, M_{\bar{\delta}}=\left.M\right|_{\delta=\bar{\delta}}=$ $\sqrt{c f(\bar{\delta}) h^{\prime}(\bar{\delta}) / h(\bar{\delta})}$, also $\omega(\bar{\delta}) M_{\bar{\delta}}=c f(\bar{\delta}) h^{\prime}(\bar{\delta})$.

At $(0,0, \bar{\delta})$, we have by simple calculation that

$$
\begin{gathered}
F_{u u u}^{1}+F_{u u v}^{2}=6 A_{30}+2 B_{21}=6\left(b_{30}-a_{30}\right)+2 c a_{21}, \\
F_{u v}^{1} F_{u u}^{1}=2 A_{11} A_{20}=-2\left(b_{20}-a_{20}\right) a_{11} M_{\bar{\delta}}, \\
F_{u v}^{2} F_{u u}^{2}=2 B_{20} B_{11}=\frac{2 c^{2}}{M_{\bar{\delta}}} a_{20} a_{11}, \\
F_{u u}^{1} F_{u u}^{2}=4 A_{20} B_{20}=\frac{4 c}{M_{\bar{\delta}}} a_{20}\left(b_{20}-a_{20}\right) .
\end{gathered}
$$

Now

$$
\begin{aligned}
a(\bar{\delta})= & \frac{1}{16}\left[F_{u u u}^{1}+F_{u u v}^{2}\right] \\
& +\frac{1}{16 \omega(\bar{\delta})}\left[F_{u v}^{1} F_{u u}^{1}-F_{u v}^{2} F_{u u}^{2}-F_{u u}^{1} F_{u u}^{2}\right] \\
= & \frac{1}{16}\left[6\left(b_{30}-a_{30}\right)+2 c a_{21}\right] \\
& +\frac{1}{16 \omega(\bar{\delta})}\left[-2\left(b_{20}-a_{20}\right) a_{11} M_{\bar{\delta}}-\frac{2 c^{2}}{M_{\bar{\delta}}} a_{11} a_{20}\right. \\
= & \frac{1}{16}\left[(f h)^{\prime \prime \prime}(\bar{\delta})-h^{\prime \prime \prime}(\bar{\delta}) f(\bar{\delta})+c h^{\prime \prime}(\bar{\delta})\right] \\
& \left.+\frac{1}{16 c f(\bar{\delta}) h^{\prime}(\bar{\delta})} a_{20}\left(b_{20}-a_{20}\right)\right] \\
& \times\left[\left\{h^{\prime \prime}(\bar{\delta}) f(\bar{\delta})-(f g)^{\prime \prime}(\bar{\delta})\right\} \frac{c f(\bar{\delta})\left\{h^{\prime}(\bar{\delta})\right\}^{2}}{h(\bar{\delta})}\right.
\end{aligned}
$$

$$
\begin{gathered}
-c^{2} f(\bar{\delta}) h^{\prime}(\bar{\delta}) h^{\prime \prime}(\bar{\delta}) \\
\left.-c f(\bar{\delta}) h^{\prime \prime}(\bar{\delta})\left\{(f h)^{\prime \prime}(\bar{\delta})-h^{\prime \prime}(\bar{\delta}) f(\bar{\delta})\right\}\right] \\
=\frac{1}{16}\left[(f h)^{\prime \prime \prime}(\bar{\delta})-h^{\prime \prime \prime}(\bar{\delta}) f(\bar{\delta})\right] \\
+\frac{1}{16}\left[\left\{h^{\prime \prime}(\bar{\delta}) f(\bar{\delta})-(f g)^{\prime \prime}(\bar{\delta})\right\}\right. \\
\left.\times \frac{\left\{\left\{h^{\prime}(\bar{\delta})\right\}^{2}+h(\bar{\delta}) h^{\prime \prime}(\bar{\delta})\right\}}{h(\bar{\delta}) h^{\prime}(\bar{\delta})}\right] \\
=\frac{1}{16 h^{\prime}(\bar{\delta})}\left[f^{\prime \prime \prime}(\bar{\delta}) h(\bar{\delta}) h^{\prime}(\bar{\delta})+2\left\{h^{\prime}(\bar{\delta})\right\}^{2} f^{\prime \prime}(\bar{\delta})\right. \\
\left.-f^{\prime \prime}(\bar{\delta}) h^{\prime \prime}(\bar{\delta}) h(\bar{\delta})\right]
\end{gathered}
$$

since $f^{\prime}(\bar{\delta})=0$.

\section{References}

[1] S. Zhang, L. Dong, and L. Chen, "The study of predator-prey system with defensive ability of prey and impulsive perturbations on the predator," Chaos, Solitons and Fractals, vol. 23, no. 2, pp. 631-643, 2005.

[2] C. S. Holling, "The functional response of predator to prey density and its role mimicry and population regulation," Memoirs of the Entomological Society of Canada, vol. 45, pp. 3-60, 1965.

[3] C. W. Clark, Bioeconomic Modelling and Fisheries Management, Wiley, New York, NY, USA, 1985.

[4] C. W. Clark, Mathematical Bioeconomics: The Optimal Management of Renewable Resource, John Wiley and Sons, New York, NY, USA, 2nd edition, 1990.

[5] D. Xiao, W. Li, and M. Han, "Dynamics in a ratio-dependent predator-prey model with predator harvesting," Journal of Mathematical Analysis and Applications, vol. 324, no. 1, pp. 1429, 2006.

[6] B. Leard, C. Lewis, and J. Rebaza, "Dynamics of ratio-dependent predator prey models with nonconstant harvesting," Discrete and Continuous Dynamical Systems, Series S, vol. 1, pp. 303-315, 2008.

[7] J. Xia, Z. Liu, R. Yuan, and S. Ruan, “The effects of harvesting and time delay on predator-prey systems with Holling type II functional response," SIAM Journal on Applied Mathematics, vol. 70, no. 4, pp. 1178-1200, 2009.

[8] U. K. Pahari and T. K. Kar, "Conservation of a resource based fishery through optimal taxation," Nonlinear Dynamics, vol. 72, pp. 591-603, 2013.

[9] R. J. Taylor, Predation, Chapman and Hall, New York, NY, USA, 1984.

[10] J. N. McNair, "The effects of refuges on predator-prey interactions: a reconsideration," Theoretical Population Biology, vol. 29, no. 1, pp. 38-63, 1986. 
[11] J. N. McNair, "Stability effects of prey refuges with entry-exit dynamics," Journal of Theoretical Biology, vol. 125, no. 4, pp. 449-464, 1987.

[12] T. K. Kar, "Modelling and analysis of a harvested prey-predator system incorporating a prey refuge," Journal of Computational and Applied Mathematics, vol. 185, no. 1, pp. 19-33, 2006.

[13] H. Wang, W. Morrison, A. Singh, and H. Weiss, "Modeling inverted biomass pyramids and refuges in ecosystems," Ecological Modelling, vol. 220, no. 11, pp. 1376-1382, 2009.

[14] L. Ji and C. Wu, "Qualitative analysis of a predator-prey model with constant-rate prey harvesting incorporating a constant prey refuge," Nonlinear Analysis: Real World Applications, vol. 11, no. 4, pp. 2285-2295, 2010.

[15] Y. Huang, F. Chen, and L. Zhong, "Stability analysis of a preypredator model with holling type III response function incorporating a prey refuge," Applied Mathematics and Computation, vol. 182, no. 1, pp. 672-683, 2006.

[16] J. Wang and L. Pan, "Qualitative analysis of a harvested predator-prey system with Holling-type III functional response incorporating a prey refuge," Advances in Difference Equations, vol. 96, pp. 1-14, 2012.

[17] T. K. Kar, A. Ghorai, and S. Jana, "Dynamics consequences of prey refuges in a two predator one prey system," Journal of Biological Systems, vol. 21, no. 2, Article ID 1350013, 28 pages, 2013.

[18] E. González-Olivares and R. Ramos-Jiliberto, "Dynamic consequences of prey refuges in a simple model system: more prey, fewer predators and enhanced stability," Ecological Modelling, vol. 166, no. 1-2, pp. 135-146, 2003.

[19] L. Chen, F. Chen, and L. Chen, "Qualitative analysis of a predator-prey model with Holling type II functional response incorporating a constant prey refuge," Nonlinear Analysis: Real World Applications, vol. 11, no. 1, pp. 246-252, 2010.

[20] G. Birkoff and G. C. Rota, Ordinary Differential Equations, Ginn, Cambridge, UK, 1982.

[21] Y. Kuang and H. I. Freedman, "Uniqueness of limit cycles in Gause-type models of predator-prey systems," Mathematical Biosciences, vol. 88, no. 1, pp. 67-84, 1988.

[22] J. Wang, J. Shi, and J. Wei, "Predator-prey system with strong Allee effect in prey," Journal of Mathematical Biology, vol. 62, no. 3, pp. 291-331, 2011.

[23] S. Wiggins, Introduction to Applied Nonlinear Dynamical Systems Chaos, Texts in Applied Mathematics, Springer, New York, NY, USA, 2nd edition, 2003.

[24] B. D. Hassard, N. D. Kazarinoff, and Y. Wan, Theory and Applications of Hopf Bifurcation, vol. 41 of London Mathematical Society Lecture Note Series, Cambridge University Press, Cambridge, UK, 1981.

[25] Y. A. Kuznetsov, Elements of Applied Bifurcation Theory, vol. 112 of Applied Mathematical Sciences, Springer, New York, NY, USA, 2004. 


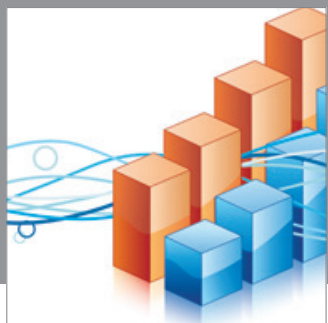

Advances in

Operations Research

mansans

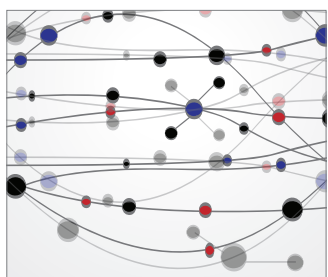

The Scientific World Journal
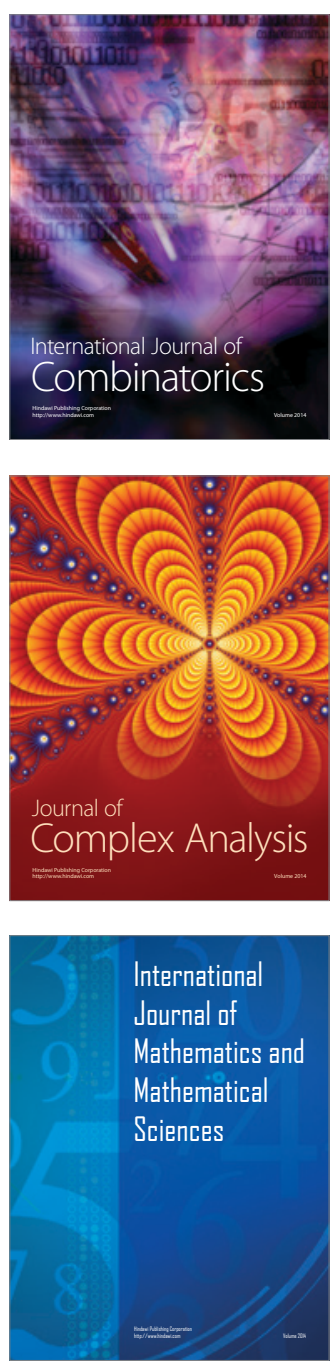
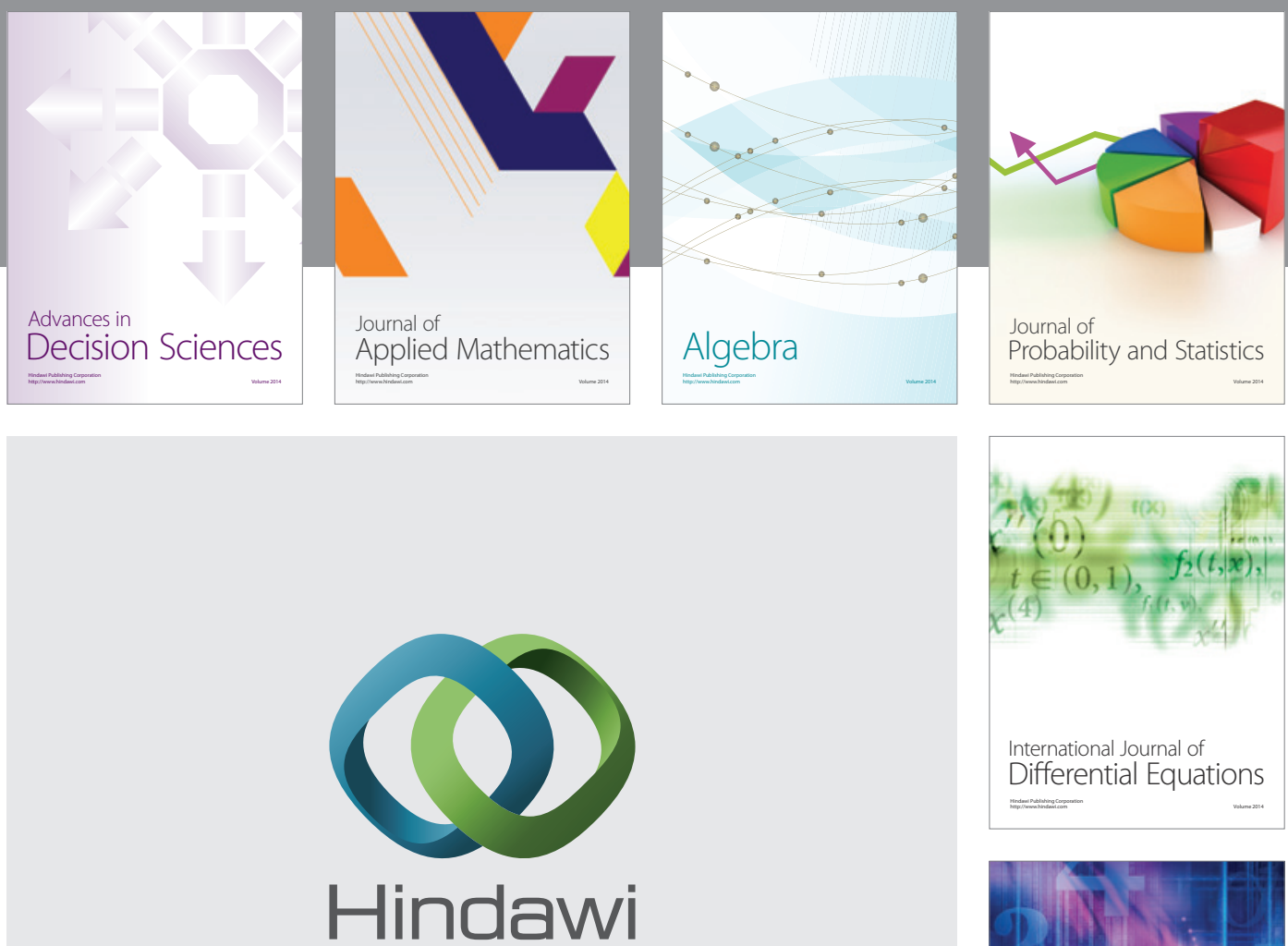

Submit your manuscripts at http://www.hindawi.com
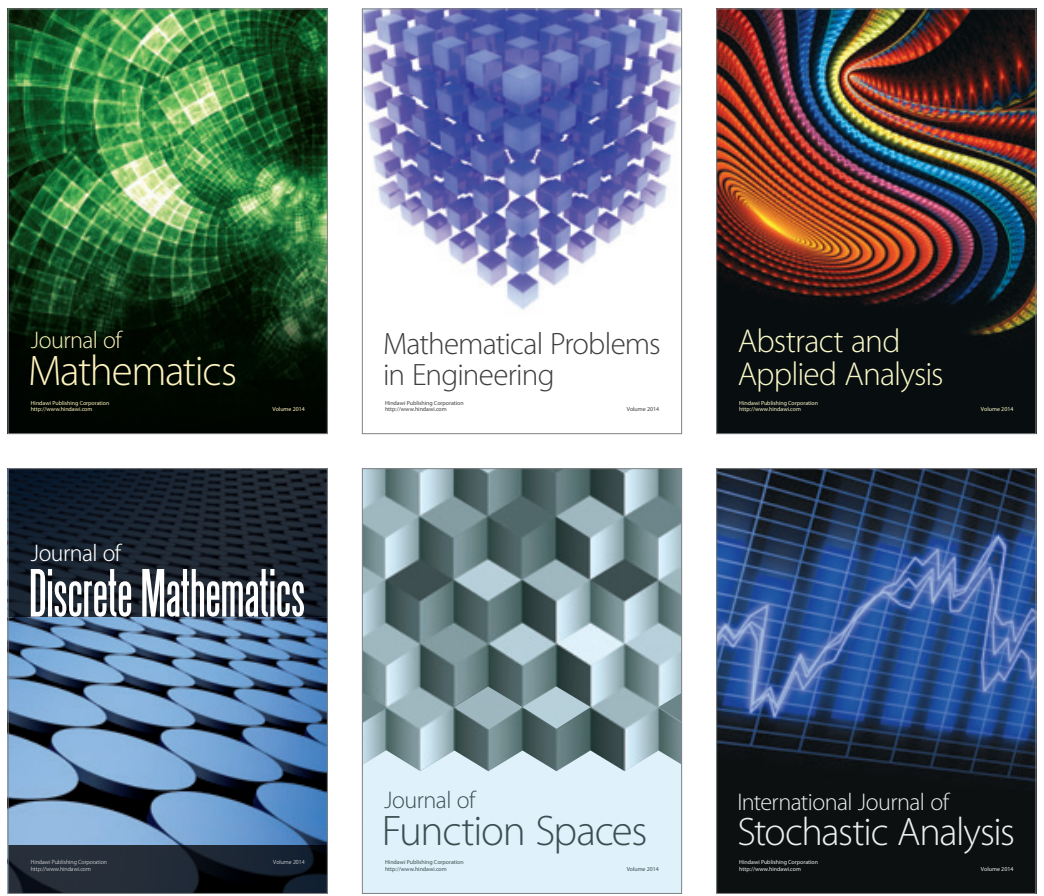

Journal of

Function Spaces

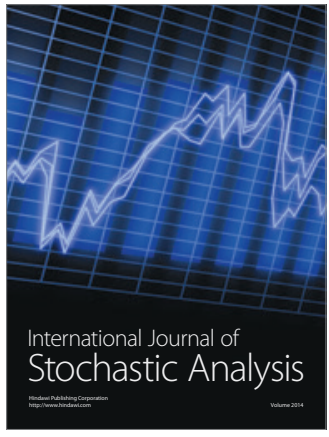

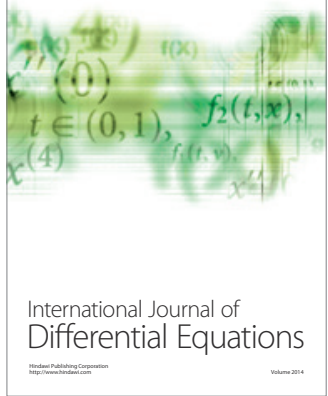
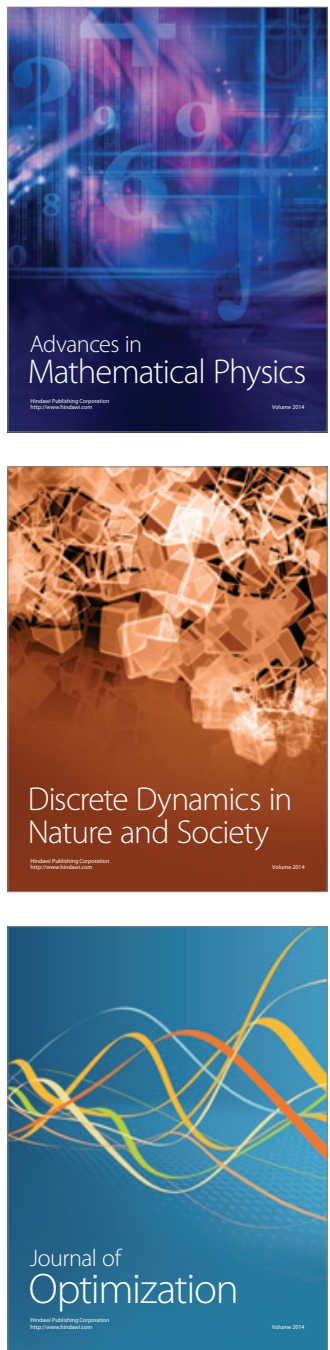